\title{
A METHOD FOR DEFECT DETECTION OF YARN-DYED FABRIC BASED ON FREQUENCY DOMAIN FILTERING AND SIMILARITY MEASUREMENT
}

\author{
Bo Zhang ${ }^{1,2}$, Chunming Tang ${ }^{1}$ \\ 1School of Electronics and Information Engineering, Tianjin Polytechnic University, Tianjin 300387, China \\ 2School of Computer and Information Engineering, Tianjin Normal University, Tianjin 300387, China \\ E-mail:tjnuzhangbo@163.com
}

\begin{abstract}
:
The detection of defects in yarn-dyed fabric is one of the most difficult problems among the present fabric defect detection methods. The difficulty lies in how to properly separate patterns, textures, and defects in the yarn-dyed fabric. In this paper, a novel automatic detection algorithm is presented based on frequency domain filtering and similarity measurement. First, the separation of the pattern and yarn texture structure of the fabric is achieved by frequency domain filtering technology. Subsequently, segmentation of the periodic units of the pattern is achieved by using distance matching function to measure the fabric pattern. Finally, based on the similarity measurement technology, the pattern's periodic unit is classified, and thus, automatic detection of the defects in the yarn-dyed fabric is accomplished.
\end{abstract}

\section{Keywords:}

yarn-dyed fabric, defect detection, frequency domain filtering, distance matching function, similarity measurement

\section{Introduction}

Fabric quality is the key edge for textile enterprises to stand out in the market amid competition, and fabric defect is an important factor affecting the quality of textiles [1]. At present, manual detection is the common method used for detection of fabric defect, but it has the disadvantages of low speed and high misdetection rates; moreover, with the increase of labor cost, its economic costs are increasing [2]. With the development of digital image processing technology since the 1970s, machine vision has been widely used in industry, and it provides a new method and means for automatic inspection of fabric defects. So far, the defect detection result of gray fabric has been satisfying [3-4]. However, the detection of defects in patterned fabric and yarn-dyed fabric is still a difficult problem in the current fabric defect detection scenario; therefore, it has become a research hotspot in the past 10 years [5-6].

The multilayered structure of yarn-dyed fabrics is the main cause for the difficulty faced during defect detection. Compared with the two-layered structure, comprising fabric texture and fabric defects, of gray fabric, there are three layers, i.e., pattern, fabric texture, and fabric defect, in yarn-dyed fabric.

In recent years, research on the defect detection of yarndyed fabric has been limited. Zhang et al. [7] focus on defect classification based on the artificial neural network, which uses morphological filtering to extract fabric defects. To deal with the problem of size selection of the structural elements in morphological filtering [8], the genetic algorithm is used to find the optimal size, and it has yielded satisfying results. However, the use of genetic algorithm increases the computation time of the system. Li et al. [9] have used log-Gabor filter and energy-based local binary patterns to extract defects and have attained good results for yarn-dyed fabric defect detection. However, the selection of parameters during the use of the logGabor filter has a great influence on the result, whereas the filter parameters obtained merely based on experience lack objectiveness. Furthermore, the size selection of the window in the local binary pattern (LBP) detection method is of equal importance, which requires the training of a defect-free image in advance. Zhu et al. [10] have used the autocorrelation function and gray-level co-occurrence matrix (GLCM) to extract the defects of yarn-dyed fabric; however, the inherent disadvantage of GLCM in texture feature extraction is that it is computationally intensive.

Due to the limitations of previous research, the existing algorithms cannot completely separate the three different structures in the fabric: patterns, fabric textures, and defects. This paper presents an automatic detection method for yarndyed fabric defects based on frequency domain filtering, distance matching function, and similarity measurement. The algorithm flow is shown in Figure 1.

The gray image with defects can be considered as the superposition of pattern $u$, fabric texture $v$, and defects $w$, as shown in Equation 1.

$$
f=u+v+w
$$

\section{Methods}

\subsection{Separation of two types of textures}

The texture background of the yarn-dyed fabric is more complicated than that of the gray fabric, because the texture 
background of the yarn-dyed fabric is the overlay of fabric pattern and fabric texture. Thus, in order to achieve the extraction of fabric defects, the separation of fabric texture and pattern texture must be accomplished first.

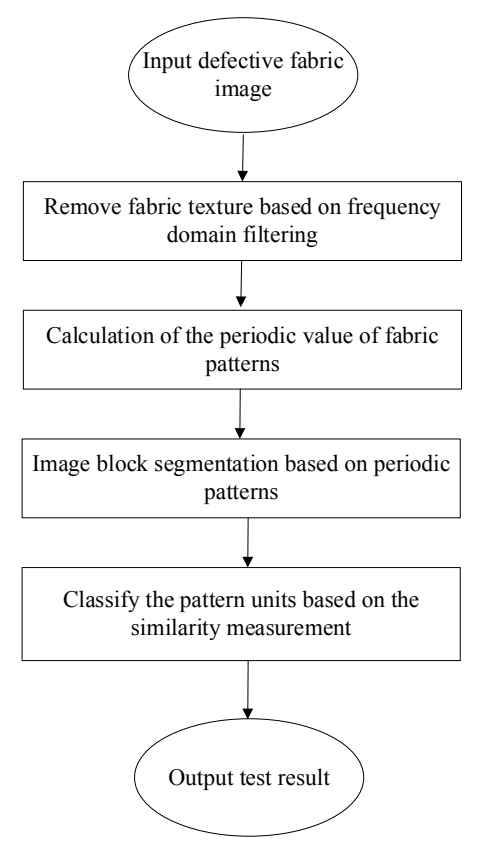

Figure 1. Block diagram of the system.

As patterns and fabric warp yarns are both periodic textures, the frequency of the two textures is different according to the time-frequency transform theory [11].

In order to achieve the separation of the two kinds of texture structures, first, we use the two-dimensional discrete Fourier transform to get the amplitude spectrum of the image. The amplitude spectrum obtained after the logarithmic transformation of the amplitude and the centralization of the spectral zeros is shown in Figure 2(b).

The longitudinal patterns of the fabric are mainly shown as a horizontal bright line in the center of the amplitude spectrum, while the transverse patterns are mainly shown as a vertical bright line in the amplitude spectrum center. In order to get the pattern information of the fabric, we only need to select the area of the horizontal bright line and the vertical bright line in the frequency domain and then filter out the other areas.

In this paper, we choose two pixels from the top and bottom of the horizontal centerline and the vertical centerline in the amplitude spectrum to form the filter template. The pixel value in the filter template is set to 1 , and the pixel value in the remaining positions is set to 0 . The filter template is shown in Figure 2(c). The filtered spectrum is reconstructed by frequency shift, exponential transformation, and Fourier inversion transform, as shown in Figure 2(d). Compared with the original figure, the pattern of different colors of the yarn is preserved, and the yarn details are filtered out.

\subsection{Dimension measurement of the periodic unit}

The fabric containing regular patterns is composed of several fabric periodic units. The shape, size, and texture intensity of the periodic units are regular in vertical and horizontal vision. Therefore, the image of yarn-dyed fabric can be considered to be composed of several periodic units.

In this paper, a distance matching function is used to calculate the pattern period in the yarn-dyed fabric [12-13].The distance matching function determines the period of fabric patterns by measuring the relationship between two elements in a regular fabric. The fabric distance matching function gets the value of the fabric period by the second-order difference operation. The fabric pattern has a strong periodicity, so the distance matching function of the fabric is periodic and diminishing. The first-order difference of the result of the distance matching function describes the change trend of the result of the distance matching function. The fastest changing position of first-order forward difference is automatically obtained by the secondorder forward difference. The periodicity and decrement of the distance matching function make the maximum of the secondorder difference correspond to the first period of the fabric, which is the period of the fabric pattern.

One-dimensional distance matching function is defined as in Equation 2.

$$
\lambda(p)=\sum_{i=1}^{N-p}[g(i)-g(i+p)]^{2}
$$

where $P$ represents the period of the function, its range of values is $(1, N-1)$, and $g(i)$ represents the function value of the function $g$ at the independent variable $i$.

Two-dimensional distance matching function of the fabric is shown in Equation 3.

$$
\left\{\begin{array}{l}
\operatorname{sum}_{x}(p)=\sum_{x=1}^{M} \sum_{y=1}^{N-p}[f(x, y)-f(x, y+p)]^{2} \\
\operatorname{sum}_{y}(p)=\sum_{y=1}^{N} \sum_{x=1}^{M-p}[f(x, y)-f(x+p, y)]^{2},
\end{array}\right.
$$

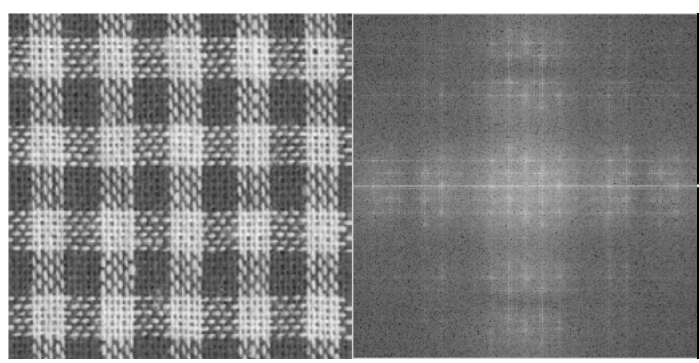

$\begin{array}{llll}(\mathrm{a}) & (\mathrm{b}) & (\mathrm{c}) & (\mathrm{d})\end{array}$

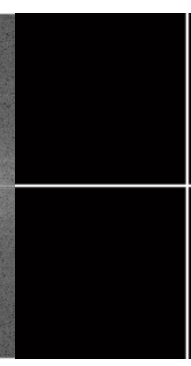

(c)

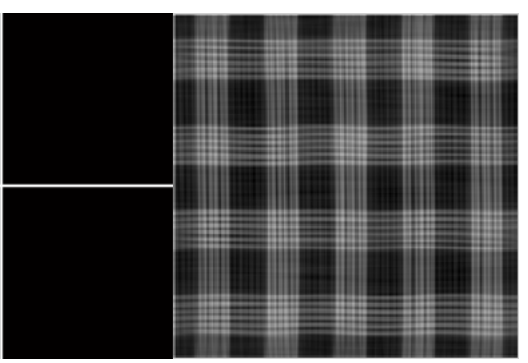

(d) 
where $\operatorname{sum}_{x}(p), \operatorname{sum}_{y}(p)$ represent the horizontal and vertical two-dimensional distance matching functions, respectively, $f(x, y)$ represents the gray value of the coordinate $(x, y)$ in the image, and $M, N$ represent the horizontal and longitudinal dimensions of the image.

The difference between the two-dimensional distance matching function corresponding to $p+1$ and the two-dimensional distance matching function corresponding to $p$ is defined as the first-order difference $D_{1}(p)$. They are the horizontal first-order difference and the vertical first-order difference, as shown in Equation 4.

$$
\left\{\begin{array}{l}
D_{1 x}(p)=\operatorname{sum}_{x}(p+1)-\operatorname{sum}_{x}(p) \\
D_{1 y}(p)=\operatorname{sum}_{y}(p+1)-\operatorname{sum}_{y}(p)
\end{array} .\right.
$$

The second-order forward difference of the horizontal and vertical directions are defined as in Equation 5.

$$
\left\{\begin{array}{l}
D_{2 x}(p)=D_{1 x}(p+1)-D_{1 x}(p) \\
D_{2 y}(p)=D_{1 y}(p+1)-D_{1 y}(p)
\end{array}\right. \text {. }
$$

We take an example of a checked cloth with pixel size of $264 \times 370$ (Figure 3 ). There are five periods in the horizontal direction, and four periods in the vertical direction; therefore, the size of its repeating element is $66 \times 74$. The results of the experiment, as shown in Figure 4, show that the experimental results are in full agreement with the artificial detection results.

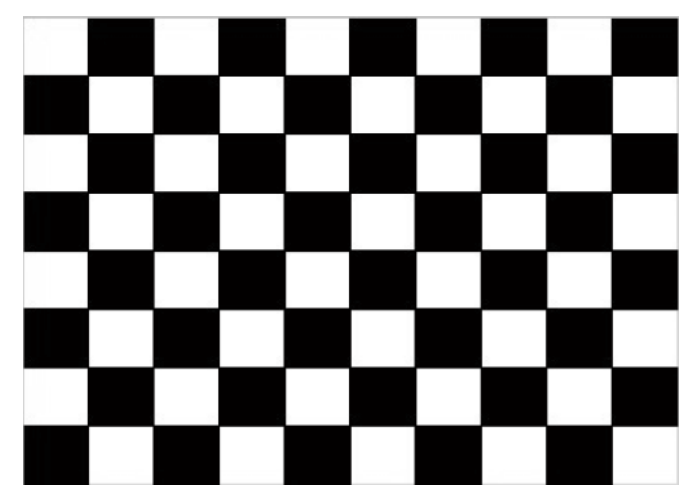

Figure 3. A typical grayscale image of a checked cloth.
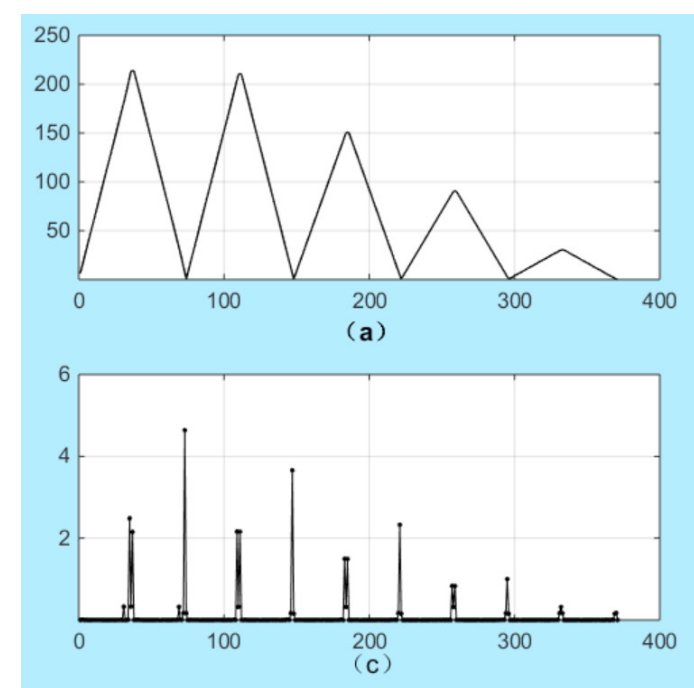

Figure 4. Period detection results of checked cloth: (a) Horizontal direction first-order difference $\left(D_{1 x}(p) / 10^{7}\right)$; (b) Vertical direction first-order difference $\left(D_{1 y}(p) / 10^{7}\right)$; (c) Horizontal direction second-order difference $\left(D_{2 x}(p) / 10^{7}\right)$; and $(d)$ Vertical direction second-order difference $\left(D_{2 y}(p) / 10^{7}\right)$.

\subsection{Classification of the periodic units based on similarity measurement}

After obtaining the periodic value of the fabric pattern, the yarn-dyed fabric is divided according to the period size. The segmented periodic units fall into two categories: defect-free and defective. The color distribution and structure of two defectfree units are almost same in the fabric, while the defective periodic unit will be different from the defect-free unit in these two aspects.

Similarity measurement can be used to distinguish the defective and defect-free regions [14] in a patterned texture. The algorithm used for defect detection in this paper is the similarity measurement of a statistical histogram of periodic units. At present, many characteristics are used in the measurement of similarity, including normalized histogram intersection coefficient, Bhattacharyya coefficient, Pearson product-moment correlation coefficient, Jaccard coefficient, and cosine angle coefficient.

In this paper, the histogram intersection coefficient is selected as the clustering feature. It is used to calculate the number of pixels with the same gray value between the two histograms. If $p$ and $q$ are two gray value distributions of images $\mathrm{A}$ and $\mathrm{B}$, and the gray value range is $i=1,2, \ldots, N$, then Equation 6 defines the histogram intersection coefficient of the two images.

$$
S_{\text {Hist }}(A, B)=\sum_{i=1}^{N} \min (p(i), q(i)) .
$$

The range of the normalized histogram intersection coefficient is $[0,1]$. Here, the numeral one indicates that the gray value of the two images is strictly matched, and zero indicates that it is completely unmatched. Suppose that the size of the periodic
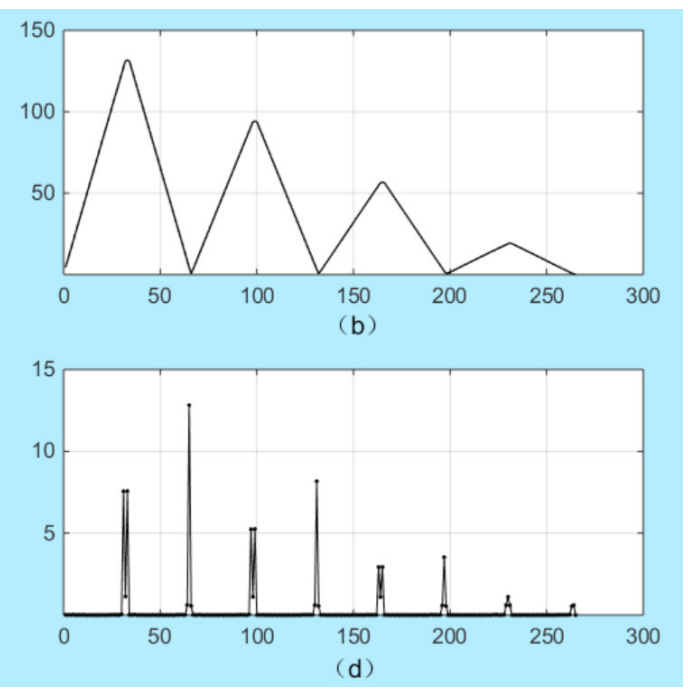

Figure 4. Period detection results of checked cloth: (a) Horizontal direction first-order difference $\left(\mathrm{D}_{1 \times(\mathrm{p})} / 10^{7}\right)$; (b) Vertical direction first-order difference $\left(D_{1 y(p)} / 10^{7}\right) ;(c)$ Horizontal direction second-order difference $\left(D_{2 \times(p)} / 10^{7}\right)$; and $(d)$ Vertical direction second-order difference $\left(D_{1 y(p)} / 10^{7}\right)$. 


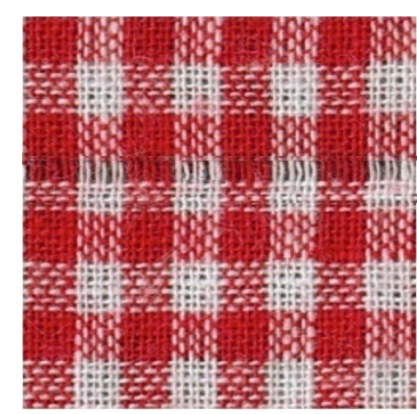

(a)

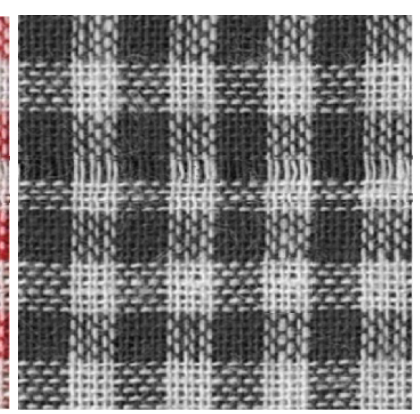

(b)

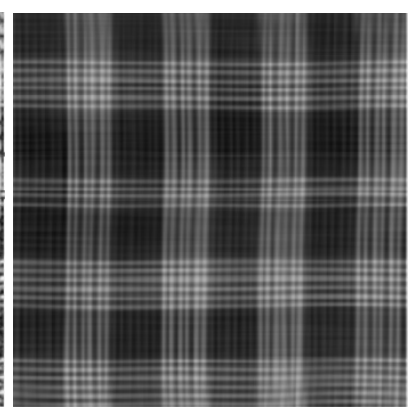

(c)

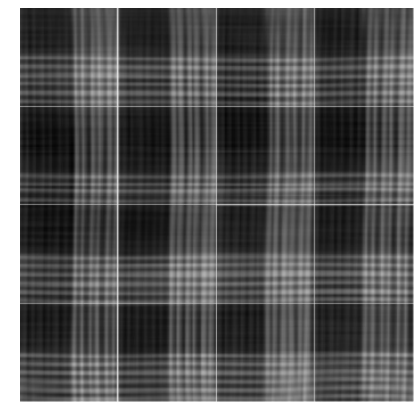

(d)

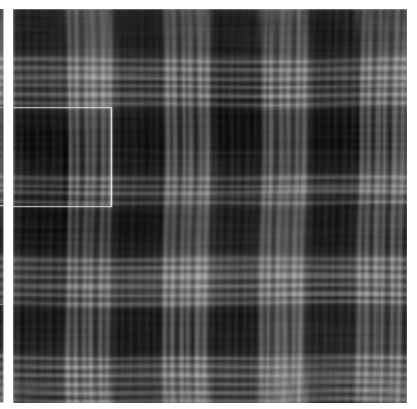

(e)

Figure 5. Defect detection results of weft-lacking: (a) color image, (b) grayscale image, (c) image after removing fabric texture, (d) image segmentation result, and (d) defect detection result.

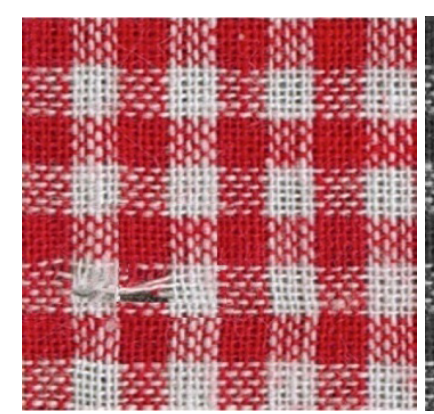

(a)

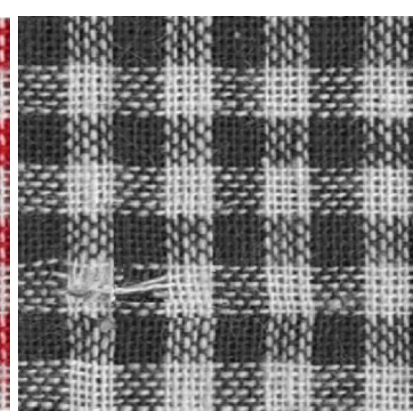

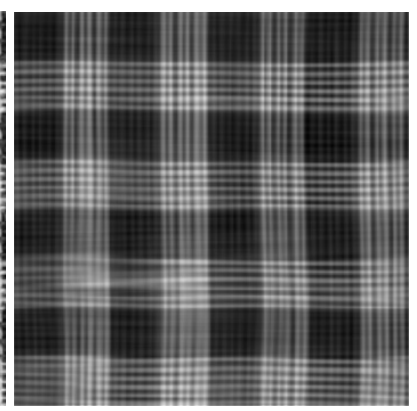

(b)

(c)

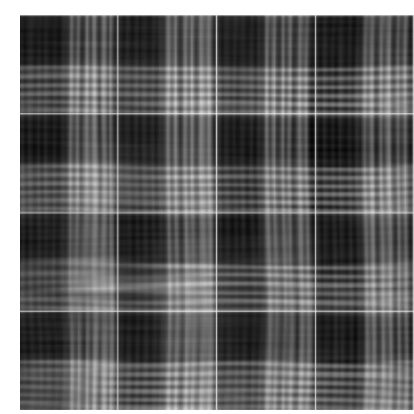

(d)

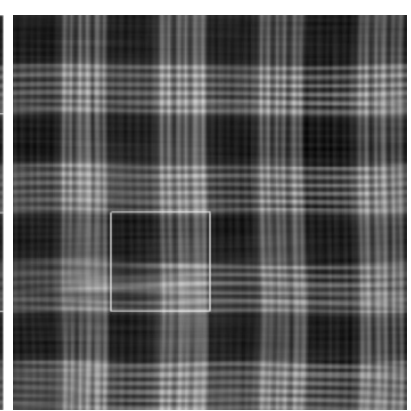

(e)

Figure 6. Defect detection results of holes: (a) color image; (b) grayscale image; (c) image after removing fabric texture; (d) image segmentation result; and (d) defect detection result.

unit obtained by the segmentation is $P_{r} \times P_{c}$; then row period $P_{r}$ represents the number of columns in a periodic unit, and the column period $P_{c}$ is the number of rows in a periodic unit.

For an image containing $n$ periodic units, the similarity matrix is obtained by Equation 7, whose size is $n \times n$. Here, $n$ is defined by Equation 8, where $M * N$ represents the size of the detected image.

$$
T_{H i s t}=\left[\begin{array}{ccccc}
S_{1,1} & S_{1,2} & \cdots & S_{1, n-1} & S_{1, n} \\
S_{2,1} & S_{2,2} & \cdots & S_{2, n-1} & S_{2, n} \\
\vdots & \vdots & S_{i, j} & \vdots & \vdots \\
S_{n-1,1} & S_{n-1,2} & \cdots & S_{n-1, n-1} & S_{n-1, n} \\
S_{n, 1} & S_{n, 2} & \cdots & S_{n, n-1} & S_{n, n}
\end{array}\right]
$$




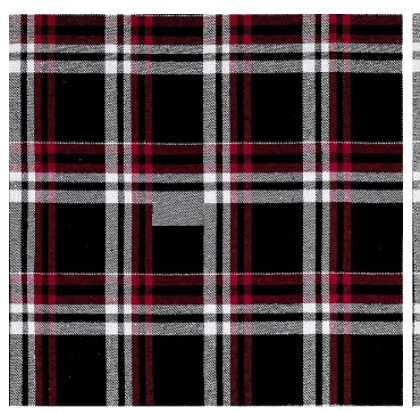

(a)

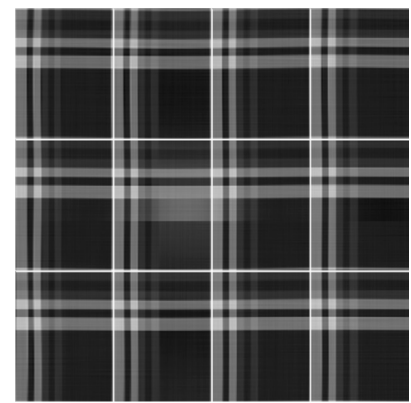

(d)

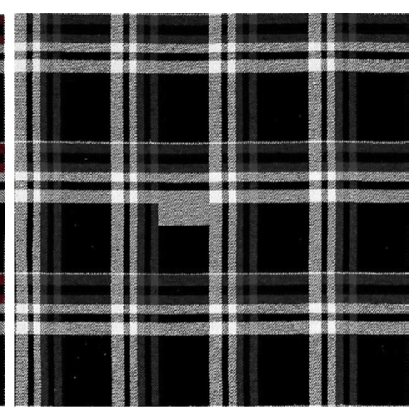

(b)

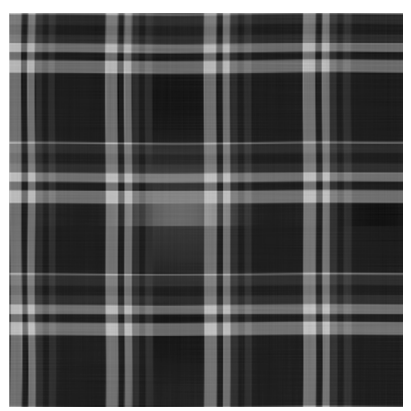

(c)

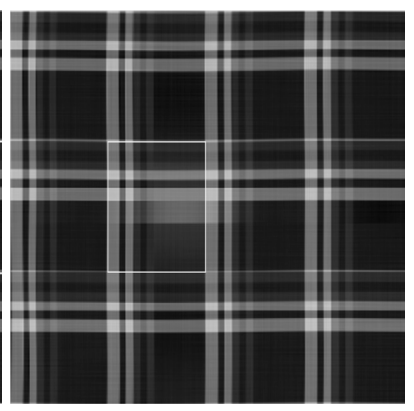

(e)

Figure 7. Detection results of an artificial defect: (a) color image; (b) grayscale image; (c) image after removing fabric texture; (d) image segmentation result; and (d) defect detection result.

$$
n=\frac{M^{*} N}{P_{r} * P_{c}}
$$

of obtaining the periodic unit dimension parameters. Suppose the size of the image is $M * N$, the value of the parameter should be selected from $M / L$ or $N / L \quad(L=2,3,4,5 \ldots$, the number of periodic units in a horizontal or vertical direction),

In Equation 7, $S_{i, j}$ represents the normalized histogram intersection coefficient between the ith periodic unit and the $j$ th periodic unit. Because the element $S_{i, j}$ in the similarity matrix is equal to the element $\mathrm{S}_{j, i}$, the matrix $T_{\text {hist }}$ is a symmetric matrix. After obtaining the similarity matrix, the average-value matrix $T_{\text {ave }}$ can be obtained, according to Equation (9), which is used to calculate the average value of each column in the similarity matrix. By setting an appropriate threshold, the location of the periodic unit with defect can be obtained by extracting the address of the column that is less than the threshold in the average-value matrix $T_{\text {ave }}$.

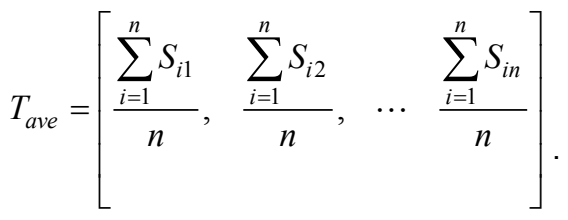

\section{Experimental results and analysis}

The most difficult detection of yarn-dyed fabric is the defect caused by the lack of warp or weft. Figure 5(a) shows the weft-lacking defect, while panels $(b-e)$ in Figure 5 show the detection process and the result. In Figure 6 , the detection process of another common defect - holes - and the results are shown. The defect in Figure 7 is manually added to test the effect of the algorithm on different fabric patterns.

In the experiment, in order to achieve average segmentation of the image, an approximate method is needed in the process

the nearest integer value, in order to split the image evenly.

\section{CONCLUSIONS}

In this study, first, the frequency filtering method is used to separate the fabric texture from the pattern texture. Then, the distance matching function is applied to measure the period value of the periodic unit. Finally, the similarity coefficient is used to discern defect-free units and defective units. Through the tests of the algorithm, this method can detect different types of defects in different kinds of patterns; moreover, the detection speed is fast and the accuracy is reliable.

\section{ACKNOWLEDGMENT}

This research was supported by the Project of Tianjin Higher Educational Science and Technology Program (2017KJ119).

\section{References}

[1] Kumar, A. (2008). Computer vision-based fabric defect detection: a survey. IEEE Transactions on Industrial Electronics, 55(1), 348-363.

[2] Ngan, H. Y. T., Pang, G. K. H., Yung N. H. C. (2011). Automated fabric defect detection-A review. Image And Vision Computing, 29(7), 442-458. 
[3] Bodnarova, A., Bennamoun, M., Latham, S. (2002). Optimal Gabor filters for textile flaw detection. Pattern Recognition, 35(12), 2973-2991.

[4] Chan, C. H., Pang, G. K. H. (2000). Fabric defect detection by Fourier analysis. IEEE Transactions on Industry Applications, 36(5), 1267-1276.

[5] Ngan, H. Y. T., Pang, G. K. H., Yung, S., Zhang, W. (2005). Wavelet based methods on patterned fabric defect detection. Pattern Recognition, 38(4), 559-576.

[6] Ng, M. K., Ngan, H. Y. T., Yuan, X. M., Zhang, W. (2014). Patterned fabric inspection and visualization by the method of image decomposition. IEEE Transactions on Automation Science and Engineering, 11(3), 943-947.

[7] Zhang, Y. H., Yuen, C. W. M., Wong, W. K., Kan, C. W. (2011). An intelligent model for detecting and classifying color-textured fabric defects using genetic algorithms and the Elman neural network. Textile Research Journal, 81(17), 1772-1787.

[8] Zhang, B., Tang, C. M. (2017). Fabric defect detection based on relative total variation model and adaptive mathematical morphology. Journal of Textile Research, 38(5), 145-149.
[9] Li, W. Y., Xue, W. L., Cheng, L. D. (2012). Intelligent detection of defects of yarn dyed fabrics by energy-based local binary patterns. Textile Research Journal, 82(19), 1960-1972.

[10] Zhu, D. D., Pan, R. R., Gao, W. D., Zhang, J. (2015). Yarn dyed fabric defect detection based on autocorrelation function and GLCM. Autex Research Journal, 15(3), 226232.

[11] Pan, R. R., Gao, W. D., Li, Z. J., Zhang, J. (2015). Woven fabric density inspection using Fourier image analysis. China Sciencepaper, 10(20), 2417-2421.

[12] Oha, G., Leeb, S. Y., Shina, S. Y. (1999). Fast determination of textural periodicity using distance matching function. Pattern Recognition Letters, 20(2), 191-197.

[13] Jing, J. F., Yang, P. P., Li, P. F. (2015). Determination on design cycle of printed fabrics based on distance matching function. Journal of Textile Research, 36(12), 98-103.

[14] Asha, V., Bhajantri, N. U., Nagabhushan, P. (2014). Similarity measures for automatic defect detection on patterned textures. International Journal of Information and Communication Technology, 4(2-4), 18-21. 